\title{
Exact solutions for two different non-linear partial differential equations
}

\author{
Durmus Daghan and R. Kubra Esen \\ Nigde Omer Halisdemir University, Department of Mathematics, Nigde, Turkey \\ Received: 6 February 2018, Accepted: 9 April 2018 \\ Published online: 30 July 2018.
}

\begin{abstract}
Travelling wave solutions of the Drinfeld-Sokolov system and Modified Benjamin-Bona-Mahony equations are studied analytically by using two dependent $\left(G^{\prime} / G, 1 / G\right)$ and $\left(1 / G^{\prime}\right)$-expansion methods. Solutions are obtained with different forms of functions as hyperbolic, trigonometric and rational functions. These methods are really effective methods, and can be simply applicable to the nonlinear evolution equations encountered in the different physical systems.
\end{abstract}

Keywords: Exact solution, $\left(G^{\prime} / G, 1 / G\right)$-expansion method.

\section{Introduction}

Mathematical solutions of different physical systems usually need non-linear differential equations. One of the these types of equations is Drinfeld-Sokolov system (DS) of partial differential equations [1,2,3]. The system was first introduced by Drinfeld and Sokolov and it is a system of nonlinear partial differential equations owner of the Lax pairs of a special form [3]. The physical motivation of this system was explained in detail by Ref.[4]. In this paper, we write the Drinfeld-Sokolov system in the following form [5],

$$
\begin{aligned}
& u_{t}+\left(v^{2}\right)_{x}=0 \\
& v_{t}-a v_{x x x}+3 b u_{x} v+3 k u v_{x}=0
\end{aligned}
$$

where $a, b$ and $k$ are arbitrary constants. The other one is Benjamin- Bona- Mahony equation (BBM) which is an alternative model for the Korteweg-de Vries equation (KdV) written by Benjamin et al. in Ref. [6], and given in the following form:

$$
u_{t}+u_{x}+u u_{x}-u_{x x t}=0
$$

The modified versions of the Benjamin- Bona- Mahony equation (MBBM) given in Eq.2 have been studied by many authors $[7,8,9,10]$. MBBM equation can also be used for the solution of different physical systems, such as acousticgravity waves in compressible fluids which are associated with variation in the background values of physical and fluid variables, acoustic waves in enharmonic crystals which are excited from the interactions of molecules, the hydromagnetic waves in cold plasma [11],[12], etc. The existence and uniqueness of the solution of initial value problems for the MBBM equation have been considered in Ref.[13]. In Ref.[9], Aslan has used the MBBM equation in the following form:

$$
u_{t}+\alpha u_{x}+\beta u^{2} u_{x}-\gamma u_{x x t}=0,
$$

\footnotetext{
* Corresponding author e-mail: durmusdaghan@ohu.edu.tr
} 
where $\alpha, \beta$ and $\gamma$ are arbitrary real constants.

In this paper, we will use the two-variable $\left(G^{\prime} / G, 1 / G\right)$-expansion and $\left(1 / G^{\prime}\right)$-expansion methods to obtain exact solutions of the DS and MBBM equations. $\left(G^{\prime} / G, 1 / G\right)$-expansion method can be considered as a generalization of the original $\left(G^{\prime} / G\right)$-expansion method [14]. As a pioneer work $\mathrm{Li}$ et al. [15] has applied the two-variable $\left(G^{\prime} / G, 1 / G\right)$-expansion method and found the exact solutions of Zakharov equations. Some applications of the $\left(G^{\prime} / G, 1 / G\right)$-expansion method can be seen in $[16,17,18,19,20] .\left(1 / G^{\prime}\right)$-expansion method introduced by Yokus [21] firstly. Some applications of the $\left(1 / G^{\prime}\right)$-expansion method can be seen in $[20,22]$.

The paper covers the following sections: in section 2 , the $\left(G^{\prime} / G, 1 / G\right)$ and $\left(1 / G^{\prime}\right)$-expansion methods to obtain exact solutions of the above equations are introduced. In Sections 3 and 4, the exact solutions of DS and MBBM equations are given, respectively. Finally, Section 5 is devoted to the conclusion of the study.

\section{Methods}

\section{$2.1\left(G^{\prime} / G, 1 / G\right)$-expansion method}

In this section, we describe the main steps of the $\left(G^{\prime} / G, 1 / G\right)$-expansion method for finding travelling wave solutions of nonlinear evolution equations. First of all, considering following second order ordinary linear differential equation:

$$
G^{\prime \prime}(\eta)+\lambda G(\eta)=\mu
$$

and defining for the simplicity

$$
\phi=G^{\prime} / G, \quad \psi=1 / G
$$

Using Eq.4 and Eq.5 yields

$$
\phi^{\prime}=-\phi^{2}+\mu \psi-\lambda, \quad \psi^{\prime}=-\phi \psi
$$

From the three cases of the general solutions of the Eq.4 we have:

Case I. When $\lambda<0$,

The general solutions of the Eq. 4 is

$$
G(\eta)=c_{1} \sinh (\sqrt{-\lambda} \eta)+c_{2} \cosh (\sqrt{-\lambda} \eta)+\frac{\mu}{\lambda}
$$

and we have

$$
\psi^{2}=-\frac{\lambda}{\lambda^{2} v+\mu^{2}}\left(\phi^{2}-2 \mu \psi+\lambda\right)
$$

where $c_{1}$ and $c_{2}$ are arbitrary constants and $v=c_{1}^{2}-c_{2}^{2}$.

Case II. When $\lambda>0$,

The general solutions of the Eq. 4 is

$$
G(\eta)=c_{1} \sin (\sqrt{\lambda} \eta)+c_{2} \cos (\sqrt{\lambda} \eta)+\frac{\mu}{\lambda}
$$


and we have

$$
\psi^{2}=\frac{\lambda}{\lambda^{2} v-\mu^{2}}\left(\phi^{2}-2 \mu \psi+\lambda\right)
$$

where $c_{1}$ and $c_{2}$ are arbitrary constants and $v=c_{1}^{2}+c_{2}^{2}$.

Case III. When $\lambda=0$,

The general solutions of the Eq. 4 is

$$
G(\eta)=\frac{\mu}{2} \eta^{2}+c_{1} \eta+c_{2}
$$

and we have

$$
\psi^{2}=\frac{1}{c_{1}^{2}-2 \mu c_{2}}\left(\phi^{2}-2 \mu \psi\right)
$$

where $c_{1}$ and $c_{2}$ are arbitrary constants.

The more details for the $\left(G^{\prime} / G, 1 / G\right)$ - expansion method can be seen in Ref.[19,20].

\section{$2.2\left(1 / G^{\prime}\right)$-expansion method}

In this section, we describe the main steps of the $\left(1 / G^{\prime}\right)$-expansion method for finding travelling wave solutions of nonlinear evolution equations. The partial differential equation (PDE)

$$
P\left(u, u_{t}, u_{x}, u_{t t}, u_{x t}, u_{x x}, \cdots\right)=0
$$

where $u=u(x, t)$ is an unknown function, $P$ is a polynomial depending on $u$ can be convert the ordinary differential equations for the transformation $\eta=x-c t$ as given below:

$$
P\left(u,-c u^{\prime}, u^{\prime}, c^{2} u^{\prime \prime},-c u^{\prime \prime}, u^{\prime \prime}, \ldots\right)=0
$$

where $u^{\prime}=\frac{d u}{d \eta}$. Suppose that the solution of Eq.11 can be expressed by a polynomial in $\left(1 / G^{\prime}\right)$

$$
u(\eta)=\sum_{i=0}^{N} a_{i}\left(\frac{1}{G^{\prime}}\right)^{i}
$$

where $G=G(\eta)$ and satisfies the following linear ordinary differential equation.

$$
G^{\prime \prime}(\eta)+\lambda G^{\prime}(\eta)+\mu=0,
$$

where $a_{i}(i=1, \ldots, N), c, \lambda$ and $\mu$ are constants to be determined later, and the positive integer $N$ can be determined by using homogeneous balance between the highest order derivatives and the nonlinear terms appearing in Eq.11. Additionally, The solution of the differential Eq. 13 is

$$
G(\eta)=c_{1} e^{-\lambda \eta}-\frac{\mu}{\lambda} \eta+c_{2},
$$

where $c_{1}$ and $c_{2}$ arbitrary integration constants. $\left(1 / G^{\prime}\right)$ term can be expressed as

$$
\left(\frac{1}{G^{\prime}}\right)=\frac{\lambda}{-\mu+\lambda c_{1}[\cosh (\lambda \eta)-\sinh (\lambda \eta)]} .
$$


The more details for the $\left(1 / G^{\prime}\right)$ - expansion method can be seen in Ref. [19].

\section{Exact solutions of DS equation}

\subsection{Application of $\left(G^{\prime} / G, 1 / G\right)$-expansion method}

DS system of equations given in Eq. 1 can be converted into following system of ordinary differential system of equations by using transformation of $\eta=x-\beta t, u=U(\eta), v=V(\eta)$,

$$
\begin{aligned}
& -\beta U^{\prime}+\left(V^{2}\right)^{\prime}=0, \\
& \beta V^{\prime}+a V^{\prime \prime \prime}-3 b U^{\prime} V-3 k U V^{\prime}=0
\end{aligned}
$$

where $U^{\prime}=\frac{d U}{d \eta}$ and $V^{\prime}=\frac{d V}{d \eta}$. The first equation given in Eq.16 can be integrated as

$$
U(\eta)=\frac{1}{\beta}\left(V(\eta)^{2}+c\right)
$$

where $\mathrm{c}$ is an arbitrary integration constant. By using the Eq.17 into Eq.16 and then integrating once, we get

$$
V^{\prime \prime}+\frac{\left(\beta^{2}-3 c k\right)}{a \beta} V-\frac{(2 b+k)}{a \beta} V^{3}+\frac{e}{a \beta}=0,
$$

where $e$ is an arbitrary integration constant [5]. Balancing the terms, $V^{3}$ and $V^{\prime \prime}$, in Eq.18, we have following form of solution:

$$
V(\eta)=a_{1} \phi+a_{0}+b_{0} \psi
$$

Substituting the Eq.19 and its derivatives into Eq.18 and using the Eqs.6 and 7, we have a set of algebraic equations for $a_{1}, a_{0}, b_{0}, k, a, b, c, e, \lambda, \mu$ and $v$ and then by solving the algebraic equations, we get

Case I. $\lambda<0$

$$
\begin{aligned}
& a_{1}= \pm \sqrt{\frac{a \beta}{4 b+2 k}}, \quad b_{0}= \pm \frac{1}{2} \sqrt{\frac{a^{2} \beta^{2} \mu^{2}+4 \beta^{4} v-24 \beta^{2} c k v+36 c^{2} k^{2} v}{\left(\beta^{2}-3 c k\right)(2 b+k)}} \\
& a_{0}=0, \quad \lambda=\frac{-2\left(\beta^{2}-3 c k\right)}{a \beta}, \quad e=0 .
\end{aligned}
$$

Substituting Eq.20 into Eq.19 we have the solution of the Eg. 18.

$$
V(\eta)= \pm \sqrt{\frac{a \beta}{4 b+2 k}} \phi \pm \frac{1}{2} \sqrt{\frac{a^{2} \beta^{2} \mu^{2}+4 \beta^{4} v-24 \beta^{2} c k v+36 c^{2} k^{2} v}{\left(\beta^{2}-3 c k\right)(2 b+k)}} \psi
$$


where $\phi$ and $\psi$ define as in Eq.5 and $G(\eta)$ define as in Eq.7. Eq.21 can be written explicitly follows:

$$
\begin{aligned}
V(\eta)= & \pm A\left(\frac{c_{1} \cosh (\sqrt{-\lambda} \eta)+c_{2} \sinh (\sqrt{-\lambda} \eta)}{c_{1} \sinh (\sqrt{-\lambda} \eta)+c_{2} \cosh (\sqrt{-\lambda} \eta)+\frac{\mu}{\lambda}}\right) \\
& \pm B\left(\frac{1}{c_{1} \sinh (\sqrt{-\lambda} \eta)+c_{2} \cosh (\sqrt{-\lambda} \eta)+\frac{\mu}{\lambda}}\right)
\end{aligned}
$$

where

$$
A=\sqrt{\frac{2\left(\beta^{2}-3 c k\right)}{4 b+2 k}}, B=\frac{1}{2} \sqrt{\frac{a^{2} \beta^{2} \mu^{2}+4 \beta^{4} v-24 \beta^{2} c k v+36 c^{2} k^{2} v}{\left(\beta^{2}-3 c k\right)(2 b+k)}}, \lambda=\frac{-2\left(\beta^{2}-3 c k\right)}{a \beta}, \quad v=c_{1}^{2}-c_{2}^{2},
$$

$c_{1}$ and $c_{2}$ are integration constants, $\eta=x-\beta t$ and $U(\eta)$ is obtained from the Eq.17.

Similarly, substituting the Eq.19 and its derivatives in Eq.18 and using the Eqs.6 and 8 yields a set of simultaneous algebraic equations for $a_{1}, a_{0}, b_{0}, k, a, b, c, e, \lambda, \mu$ and $v$ and then by solving the algebraic equations, we have,

Case II. $\lambda>0$

$$
\begin{aligned}
& a_{1}= \pm \sqrt{\frac{a \beta}{4 b+2 k}}, \quad b_{0}= \pm \frac{1}{2} \sqrt{\frac{a^{2} \beta^{2} \mu^{2}-4 \beta^{4} v+24 \beta^{2} c k v-36 c^{2} k^{2} v}{\left(\beta^{2}-3 c k\right)(2 b+k)}} \\
& a_{0}=0, \quad \lambda=\frac{-2\left(\beta^{2}-3 c k\right)}{a \beta}, \quad e=0 .
\end{aligned}
$$

Substituting Eq.23 into Eq.19 we have the solution of the Eg. 18.

$$
V(\eta)= \pm \sqrt{\frac{a \beta}{4 b+2 k}} \phi \pm \frac{1}{2} \sqrt{\frac{a^{2} \beta^{2} \mu^{2}-4 \beta^{4} v+24 \beta^{2} c k v-36 c^{2} k^{2} v}{\left(\beta^{2}-3 c k\right)(2 b+k)}} \psi
$$

where $\phi$ and $\psi$ define as in Eq.5 and $G(\eta)$ define as in Eq.8. Eq.24 can be written explicitly follows:

$$
\begin{aligned}
V(\eta)= & \pm A\left(\frac{c_{1} \cos (\sqrt{\lambda} \eta)-c_{2} \sin (\sqrt{\lambda} \eta)}{c_{1} \sin (\sqrt{\lambda} \eta)+c_{2} \cos (\sqrt{\lambda} \eta)+\frac{\mu}{\lambda}}\right) \\
& \pm B\left(\frac{1}{c_{1} \sin (\sqrt{\lambda} \eta)+c_{2} \cos (\sqrt{\lambda} \eta)+\frac{\mu}{\lambda}}\right)
\end{aligned}
$$

where

$$
A=\sqrt{\frac{-2\left(\beta^{2}-3 c k\right)}{4 b+2 k}}, B=\frac{1}{2} \sqrt{\frac{a^{2} \beta^{2} \mu^{2}-4 \beta^{4} v+24 \beta^{2} c k v-36 c^{2} k^{2} v}{\left(\beta^{2}-3 c k\right)(2 b+k)}}, \lambda=\frac{-2\left(\beta^{2}-3 c k\right)}{a \beta}, \quad v=c_{1}^{2}+c_{2}^{2},
$$

$c_{1}$ and $c_{2}$ are integration constants, $\eta=x-\beta t$ and $U(\eta)$ is obtained from the Eq.17.

The last one, substituting the Eq.19 and its derivatives in Eq.18 and using the Eqs.6 and 9, we get algebraic equations for $a_{1}, a_{0}, b_{0}, k, a, b, c, e, \mu, c_{1}$ and $c_{2}$ and then by solving the algebraic equations we arrive following solution: 
Case III. $\lambda=0$

$$
\begin{aligned}
& a_{1}= \pm \sqrt{\frac{a \beta}{4 b+2 k}}, \quad b_{0}= \pm \sqrt{\frac{-a \beta\left(2 c_{2} \mu-c_{1}^{2}\right)}{4 b+2 k}}, \quad a_{0}=0, \\
& \beta= \pm \sqrt{3 c k} \quad \lambda=0, \quad e=0 .
\end{aligned}
$$

Substituting Eq.26 into Eq.19 we have the solution of the Eg. 18.

$$
V(\eta)= \pm \sqrt{\frac{a \beta}{4 b+2 k}} \phi \pm \sqrt{\frac{-a \beta\left(2 c_{2} \mu-c_{1}^{2}\right)}{4 b+2 k}} \psi .
$$

where $\beta= \pm \sqrt{3 c k}, \phi$ and $\psi$ define as in Eq.5 and $G(\eta)$ define as in Eq.9. Eq.27 can be written explicitly follows:

$$
V(\eta)= \pm A\left(\frac{\mu \eta+c_{1}}{\frac{\mu}{2} \eta^{2}+c_{1} \eta+c_{2}}\right) \pm B\left(\frac{1}{\frac{\mu}{2} \eta^{2}+c_{1} \eta+c_{2}}\right)
$$

where

$$
A=\sqrt{\frac{a \beta}{4 b+2 k}}, \quad B=\sqrt{\frac{-a \beta\left(2 c_{2} \mu-c_{1}^{2}\right)}{4 b+2 k}}, \quad \eta=x-\beta t, \beta= \pm \sqrt{3 c k}
$$

and $U(\eta)$ is obtained from the Eq. 17.

\subsection{Application of $\left(1 / G^{\prime}\right)$-expansion method}

We use balance $N=1$ for the solution of the Eq. 18, we get the following type solution.

$$
V(\eta)=a_{0}+a_{1}\left(\frac{1}{G^{\prime}}\right)
$$

Substituting Eq.29 in Eq. 18 then collecting all coefficient with respect to $\left(1 / G^{\prime}\right)$ end equating to zero we get following system of equations.

$$
\begin{aligned}
& \left(\frac{1}{G^{\prime}}\right)^{0}: \quad-2 a_{0}^{3} b-a_{0}^{3} k+a_{0} \beta^{2}-3 a_{0} c k+e \\
& \left(\frac{1}{G^{\prime}}\right)^{1}: \quad a_{1}\left(-6 a_{0}^{2} b-3 a_{0}^{2} k+a \beta \lambda^{2}+\beta^{2}-3 c k\right), \\
& \left(\frac{1}{G^{\prime}}\right)^{2}: \quad 3 a_{1}\left(-2 a_{1} a_{0} b-a_{1} a_{0} k+a \beta \lambda \mu\right) \\
& \left(\frac{1}{G^{\prime}}\right)^{3}: \quad a_{1}\left(-2 a_{1}^{2} b-a_{1}^{2} k+2 a \beta \mu^{2}\right)
\end{aligned}
$$

Solving the system of equations above, we have

$$
\begin{aligned}
& a_{1}= \pm \sqrt{\frac{2 a \beta}{2 b+k} \mu, \quad a_{0}}= \pm \frac{1}{2} \sqrt{\frac{2 a \beta}{2 b+k}} \lambda, \\
& \lambda= \pm \sqrt{\frac{2\left(\beta^{2}-3 c k\right)}{a \beta}}, \quad e=0 .
\end{aligned}
$$


Substituting these solutions into Eq.29, we obtain following solution.

$$
V(\eta)= \pm \sqrt{\frac{2 a \beta}{2 b+k}} \mu\left(\frac{1}{G^{\prime}}\right)+\sqrt{\frac{\beta^{2}-3 c k}{2 b+k}}
$$

where

$$
\left(\frac{1}{G^{\prime}}\right)=\frac{\lambda}{-\mu+\lambda c_{1}[\cosh (\lambda \eta)-\sinh (\lambda \eta)]}, \lambda= \pm \sqrt{\frac{2\left(\beta^{2}-3 c k\right)}{a \beta}}
$$

and $c_{1}$ is arbitrary integration constant.

\section{Exact solutions of MBBM equation}

\subsection{Application of $\left(G^{\prime} / G, 1 / G\right)$-expansion method}

Eq.3 can be converted into following ordinary differential equation by using transformation of $\eta=k x+w t, u=U(\eta)$,

$$
-\gamma k^{2} w U^{\prime \prime \prime}+\beta k U^{2} U^{\prime}+(w+\alpha k) U^{\prime}=0
$$

Integration Eq.31 once, we get

$$
U^{\prime \prime}-\frac{(w+\alpha k)}{\gamma k^{2} w} U-\frac{\beta}{3 \gamma k w} U^{3}-\frac{c}{\gamma k^{2} w}=0 .
$$

where $c$ is an integration constant. Considering the homogeneous balance between the terms, $U^{3}$ and $U^{\prime \prime}$, in Eq.32, we have reached the following form of solution:

$$
U(\eta)=a_{1} \phi+a_{0}+b_{0} \psi
$$

Substituting the Eq.33 and its derivatives into Eq.32. Then, using the Eq.6 with Eqs. 7, 8 ,9, we get simultaneous algebraic equations for $a_{1}, a_{0}, b_{0}, k, w, \beta, c, \alpha, \lambda, c_{1}, \mu$ and $v$ in the cases $\lambda<0, \lambda>0$ and $\lambda=0$. Then, solving the algebraic equations for each cases, we can obtain following solutions.

Case I. $\lambda<0$

$$
\begin{array}{r}
a_{1}= \pm \sqrt{\frac{3 \gamma k w}{2 \beta}}, \quad b_{0}= \pm \frac{1}{2} \sqrt{\frac{-12 \alpha^{2} k^{2} v-24 \alpha k v w-3 \gamma^{2} k^{4} \mu^{2} w^{2}-12 v w^{2}}{\beta k(w+\alpha k)}} \\
a_{0}=0, \quad \lambda=\frac{2(w+\alpha k)}{\gamma k^{2} w}, \quad c=0 .
\end{array}
$$

Substituting Eq.34 into Eq.33 we have the solution of the Eg. 32.

$$
U(\eta)= \pm \sqrt{\frac{3 \gamma k w}{2 \beta}} \phi \pm \frac{1}{2} \sqrt{\frac{-12 \alpha^{2} k^{2} v-24 \alpha k v w-3 \gamma^{2} k^{4} \mu^{2} w^{2}-12 v w^{2}}{\beta k(w+\alpha k)}} \psi
$$


where $\phi$ and $\psi$ define as in Eq.5 and $G(\eta)$ define as in Eq.7. Eq.35 can be written explicitly follows:

$$
\begin{aligned}
U(\eta)=\quad & \pm A\left(\frac{c_{1} \cosh (\sqrt{-\lambda} \eta)+c_{2} \sinh (\sqrt{-\lambda} \eta)}{c_{1} \sinh (\sqrt{-\lambda} \eta)+c_{2} \cosh (\sqrt{-\lambda} \eta)+\frac{\mu}{\lambda}}\right) \\
& \pm B\left(\frac{1}{c_{1} \sinh (\sqrt{-\lambda} \eta)+c_{2} \cosh (\sqrt{-\lambda} \eta)+\frac{\mu}{\lambda}}\right)
\end{aligned}
$$

where

$A=\sqrt{\frac{-3(w+\alpha k)}{\beta k}}, B=\frac{1}{2} \sqrt{\frac{-12 \alpha^{2} k^{2} v-24 \alpha k v w-3 \gamma^{2} k^{4} \mu^{2} w^{2}-12 v w^{2}}{\beta k(w+\alpha k)}}, \lambda=\frac{2(w+\alpha k)}{\gamma k^{2} w}, v=c_{1}^{2}-c_{2}^{2}, \eta=k w+w t$

Case II. $\lambda>0$

$$
\begin{array}{r}
a_{1}= \pm \sqrt{\frac{3 \gamma k w}{2 \beta}}, \quad b_{0}= \pm \frac{1}{2} \sqrt{\frac{12 \alpha^{2} k^{2} v+24 \alpha k v w-3 \gamma^{2} k^{4} \mu^{2} w^{2}+12 v w^{2}}{\beta k(w+\alpha k)}} \\
a_{0}=0, \quad \lambda=\frac{2(w+\alpha k)}{\gamma k^{2} w}, \quad c=0 .
\end{array}
$$

Substituting Eq.37 into Eq.33 we have the solution of the Eg. 32.

$$
U(\eta)= \pm \sqrt{\frac{3 \gamma k w}{2 \beta}} \phi \pm \frac{1}{2} \sqrt{\frac{12 \alpha^{2} k^{2} v+24 \alpha k v w-3 \gamma^{2} k^{4} \mu^{2} w^{2}+12 v w^{2}}{\beta k(w+\alpha k)}} \psi
$$

where $\phi$ and $\psi$ define as in Eq.5 and $G(\eta)$ define as in Eq.8. Eq.38 can be written explicitly follows:

$$
\begin{aligned}
U(\eta)=\quad & \pm A\left(\frac{c_{1} \cos (\sqrt{\lambda} \eta)-c_{2} \sin (\sqrt{\lambda} \eta)}{c_{1} \sin (\sqrt{\lambda} \eta)+c_{2} \cos (\sqrt{\lambda} \eta)+\frac{\mu}{\lambda}}\right) \\
& \pm B\left(\frac{1}{c_{1} \sin (\sqrt{\lambda} \eta)+c_{2} \cos (\sqrt{\lambda} \eta)+\frac{\mu}{\lambda}}\right)
\end{aligned}
$$

where

$A=\sqrt{\frac{3(w+\alpha k)}{\beta k}}, B=\frac{1}{2} \sqrt{\frac{12 \alpha^{2} k^{2} v+24 \alpha k v w-3 \gamma^{2} k^{4} \mu^{2} w^{2}+12 v w^{2}}{\beta k(w+\alpha k)}}, \lambda=\frac{2(w+\alpha k)}{\gamma k^{2} w}, \quad v=c_{1}^{2}+c_{2}^{2}, \quad \eta=k w+w t$.

Case III. $\lambda=0$

$$
\begin{array}{r}
a_{1}= \pm \sqrt{\frac{3 \gamma w k}{2 \beta}}, \quad b_{0}= \pm \sqrt{\frac{-6 c_{2} \gamma \mu w k+3 c_{1}^{2} \gamma w k}{2 \beta}} \\
a_{0}=0, \quad w=-\alpha k, \quad \lambda=0, \quad c=0
\end{array}
$$


Substituting Eq.40 into Eq.33 we have the solution of the Eg. 32.

$$
U(\eta)= \pm \sqrt{\frac{3 \gamma w k}{2 \beta}} \phi \pm \sqrt{\frac{-6 c_{2} \gamma \mu w k+3 c_{1}^{2} \gamma w k}{2 \beta}} \psi
$$

where $w=-\alpha k, \phi$ and $\psi$ define as in Eq.5 and $G(\eta)$ define as in Eq.9. Eq.41 can be written explicitly follows:

$$
U(\eta)= \pm A\left(\frac{\mu \eta+c_{1}}{\frac{\mu}{2} \eta^{2}+c_{1} \eta+c_{2}}\right) \pm B\left(\frac{1}{\frac{\mu}{2} \eta^{2}+c_{1} \eta+c_{2}}\right)
$$

where $A=\sqrt{\frac{3 \gamma w k}{2 \beta}}, B=\sqrt{\frac{-6 c_{2} \gamma \mu w k+3 c_{1}^{2} \gamma w k}{2 \beta}}, \eta=k x+w t, w=-\alpha k$

\subsection{Application of $\left(1 / G^{\prime}\right)$-expansion method}

We use balance $N=1$ for the solution of the Eq. 32, we get the following type solution.

$$
U(\eta)=a_{0}+a_{1}\left(\frac{1}{G^{\prime}}\right)
$$

Substituting Eq.43 in Eq. 32 then collecting all coefficient with respect to $\left(1 / G^{\prime}\right)$ end equating to zero we get following system of equations.

$$
\begin{aligned}
& \left(\frac{1}{G^{\prime}}\right)^{0}: \quad a_{0}^{3}+\beta k+3 a_{0} \alpha k+3 a_{0} w+3 c \\
& \left(\frac{1}{G^{\prime}}\right)^{1}: \quad 3 a_{1}\left(a_{0}^{2} \beta k+\alpha k-\gamma k^{2} \lambda^{2} w+w\right), \\
& \left(\frac{1}{G^{\prime}}\right)^{2}: \quad 3 a_{1} k\left(a_{1} a_{0} \beta-3 \gamma k \lambda \mu w\right), \\
& \left(\frac{1}{G^{\prime}}\right)^{3}: \quad a_{1} k\left(a_{1}^{2} \beta-6 \gamma k \mu^{2} w\right),
\end{aligned}
$$

Solving the system of equations above, we have

$$
\begin{aligned}
& a_{1}= \pm \sqrt{\frac{6 \gamma k w}{\beta}} \mu, \quad a_{0}= \pm \frac{1}{2} \sqrt{\frac{6 \gamma k w}{\beta}} \lambda, \\
& \lambda= \pm \sqrt{-\frac{2(w+\alpha k)}{\gamma w}} \frac{1}{k}, \quad c=0 .
\end{aligned}
$$

Substituting these solutions into Eq.43, we obtain following solution.

$$
U(\eta)= \pm \sqrt{\frac{6 \gamma k w}{\beta}} \mu\left(\frac{1}{G^{\prime}}\right)+\sqrt{-\frac{3(w+\alpha k)}{\beta k}}
$$

where

$$
\left(\frac{1}{G^{\prime}}\right)=\frac{\lambda}{-\mu+\lambda c_{1}[\cosh (\lambda \eta)-\sinh (\lambda \eta)]}, \quad \lambda= \pm \sqrt{-\frac{2(w+\alpha k)}{\gamma w}} \frac{1}{k}
$$


and $c_{1}$ is arbitrary integration constant.

\section{Conclusion}

Drinfeld-Sokolov and Modified Benjamin-Bona-Mahony equations are studied analytically by using two dependent $\left(G^{\prime} / G, 1 / G\right)$-expansion and $\left(1 / G^{\prime}\right)$-expansion methods. We obtained exact solutions of these equations for different forms of functions as hyperbolic, trigonometric and rational functions. These two types methods can be applied to the nonlinear partial differential equations in the physical systems such as various physical and astrophysical plasma systems.

\section{Competing interests}

The authors declare that they have no competing interests.

\section{Authors' contributions}

All authors have contributed to all parts of the article. All authors read and approved the final manuscript.

\section{References}

[1] Wang,JP.: A list of $1+1$ dimensional integrable equations and their properties, J. Nonlinear Math. Phys. 9, 213-233, (2002).

[2] Olver, PJ.: Applications of lie groups to differential equations, Springer, New York, 1993.

[3] Goktas, U.Hereman, E.: Symbolic computation of conserved densities for systems of nonlinear evolution equations, J. Symb. Comput. 24(5), 591-621, (1997).

[4] Gurses, M.Karasu A.: Integrable KdV systems: Recursion operators of degree four, Phys. Lett. A. 251, 247-249, (1999).

[5] Daghan, D.Yildiz, O.Toros, S.: Comparison of $\left(G^{\prime} / G\right)$-methods for finding exact solutions of the Drinfeld-Sokolov system, Mathematica Slovaca., 65(3), 607-632,(2015).

[6] Benjamin, T.B.Bona, J.L.Mahony, J.J.: Model equations for long waves in nonlinear dispersive systems, Philos Trans. R. Soc. London, Ser. A. 272, 47-48, (1972).

[7] Wazwaz, A.M.Helal, M.A.: Nonlinear variants of the BBM equation with compact and noncompact physical structures, Chaos Solit. and Fractals. 26, 767-776, (2005).

[8] Nickel, J.: Elliptic solutions to a generalized BBM equation, Phys. Lett. A. 364(3-4), 221-226, (2007).

[9] Aslan, I.: Exact and explicit solutions to some nonlinear evolution equations by utilizing the $\left(G^{\prime} / G\right)$-expansion method, Appl. Math. and Comput. 215(2), 857-863, (2009).

[10] Layeni,O.P. Akinola, A.P.: A new hyperbolic auxiliary function method and exact solutions of the mBBM equation, Commun. Nonlinear Sci. Numer. Simul. 15(2), 135-138, (2010). Corrigendum: Nonlinear Sci. Numer. Simul. 15(9), 2734, (2010).

[11] Saut, J.C.Tzetkov, N.: Global well-posedness for the KP-BBM equations, Appl. Math. Res. Express. 1, 1-6, (2004).

[12] Varlamov, V. Liu, Y.: Cauchy problem for the Ostrovsky equation, Discrete Dynam. Syst. 10, 731-753, (2004).

[13] Tso, T.: Existence of solution of the modified Benjamin-Bona-Mahony equation, Chin. J. Math. 24(4), 327-336, (1996).

[14] Wang, M.L.Li, X.Zhang, J.: The $\left(G^{\prime} / G\right)$-Expansion Method and Traveling Wave Solutions of Nonlinear Evolution Equations in Mathematical Physics, Phys. Lett. A. 372(4), 417-423, (2008).

[15] Li,L-X.Li, E-Q.Wang, M.L.: The $\left(G^{\prime} / G, 1 / G\right)$-expansion method and its application to travelling wave solutions of the Zakharov equations, Appl. Math. A J. Chin. Univ. 25, 454-462, (2010).

[16] Zayed, E.M.E. Abdelaziz, M.A.M.: The two variables $\left(G^{\prime} / G, 1 / G\right)$-expansion method for solving the nonlinear $K d V$-mKdV equation, Math. Probl. Eng. 2012, 725061, (2012). 
[17] Zayed, E.M.E. Hoda Ibrahim, S.A. Abdelaziz, M.A.M.: Traveling wave solutions of the nonlinear 3+1-dimensional KadomtsevPetviashvili equation using the two variables $\left(G^{\prime} / G, 1 / G\right)$-expansion method, J. Appl.Math. 2012, 560531,(2012).

[18] Demiray, S. Unsal, O. Bekir, A.:: Exact solutions of nonlinear wave equations using $\left(G^{\prime} / G, 1 / G\right)$-expansion method, Journal of the Egyptian Mathematical Society, 23, 78-84, (2015).

[19] Daghan, D.Donmez, O.: Investigating the effect of integration constants and various plasma parameters on the dynamics of the soliton in different physical plasmas, Physics of Plasmas, 22, 072114, (2015).

[20] Daghan, D.Donmez, O.: Exact Solutions of the Gardner Equation and their Applications to the Different Physical Plasmas., Brazilian Journal of Physics, 46, 321-333,(2016).

[21] Yokus, A.: Ph.D. Thesis, Firat University, Elazig, 2011.

[22] Demiray, S.Unsal, O.Bekir, A.:New Exact Solutions for Boussinesq Type Equations by Using $\left(G^{\prime} / G, 1 / G\right)$ and $\left(1 / G^{\prime}\right)$-Expansion Methods, Acta Physica Polonica A, 125(5), 1093-1098, (2014). 